\title{
Male Erectile Disorder
}

National Cancer Institute

\section{Source}

National Cancer Institute. Male Erectile Disorder. NCI Thesaurus. Code C34801.

A disorder characterized by the persistent or recurrent inability to achieve or to maintain an erection during sexual activity. 